\title{
nazionale sulla storia del fascismo. Spazio di confronto
}

La volontà dell'amministrazione comunale di Predappio, paese natale di Mussolini in provincia di Forlì, di realizzare un museo/centro di interpretazione sulla storia del fascismo nella ex Casa del fascio ha suscitato e continua a suscitare un aspro dibattito pubblico, con ampi riflessi interni anche alla nostra redazione. Dato che l'ideazione del progetto scientifico è stata affidata dal Comune all'Istituto per la storia e le memorie del '900 Parri - Emilia Romagna, come rivista degli Istituti storici dell'Emilia Romagna in Rete, ovvero quale snodo di relazione e informazione on line fra gli istituti storici regionali (interni ed esterni alla rete INSMLI), sentiamo la necessità di confrontarci sul tema e aprire una riflessione pubblica su tale iniziativa.

Come già emerso dal dibattito sulla stampa cartacea e on-line l'operazione porta infatti con sè alcuni rischi tangibili: la complessità storiografica dell'oggetto "fascismo", nella sua duplice natura di soggetto modernizzatore e oppressore, contemporaneamente nazionalista e transnazionale, circoscritto a una specifica epoca storica ma con profonde radici nel tessuto politico-culturale italiano; il problema del luogo prescelto per realizzare il progetto, privo di centralità nazionale sia nell'evoluzione politico-istituzionale del regime sia nella geografia culturale e infrastrutturale dell'Italia odierna, ma al contempo meta di pellegrinaggi e "luogo di memoria" per reduci, nostalgici e sdoganatori del Ventennio fascista.

D'altro lato, sulla scia di altre esperienze europee, il museo potrebbe rappresentare invece un'opportunità per fare i conti con il fascismo, le sue colpe, le eredità 
e le sue continuità storiche, generando una proficua ricaduta della recente riflessione storiografica nella dimensione pubblica.

Se la notizia della creazione di un primo, grande museo sulla storia del fascismo in Italia ha sollevato forti critiche in ambito sia politico che storiografico - in particolare sull'opportunità della sede prescelta - solo in tempi recenti è stato possibile accedere a elementi informativi sull'approccio, gli obiettivi e le forme che il museo si prefigge concretamente di assumere. Per questo motivo la nostra prima esigenza è stata quella di raccogliere e mettere a disposizione la documentazione attualmente accessibile - progetto museografico, composizione comitato scientifico e gruppo di lavoro, atti del convegno "Narrare il fascismo" - così come quella che potrà esserlo in futuro. La conoscenza di questi materiali costituisce, a nostro giudizio, la condizione minima di partenza per poter passare da un confronto sull'opportunità di realizzare tale museo a uno sui contenuti dello stesso - vero centro della riflessione a progetto in corso - nell'intento di offrire uno sguardo analitico sulle modalità con le quali si sta attualmente configurando l'idea espositiva, nonché su altre esperienze europee che hanno affrontato analoghe problematiche.

La redazione ha quindi scelto di aprire uno spazio di libera discussione, coinvolgendo figure interne ed esterne al progetto, sui contenuti e le modalità di veicolazione e comunicazione degli stessi.

A fianco di interventi commissionati a esperti sul piano storico e museale, la rivista accoglierà proposte di riflessione in forma di testi brevi senza note $(10 / 15.000$ battute) sul contenuto dell'operazione e le modalità di realizzazione.

I contributi potranno vertere sui seguenti nuclei tematici o su altri affini, purchè pertinenti al tema.

- Criteri e approcci metodologici del progetto museografico

- Odierne narrazioni pubbliche del fascismo

- Comparazione con altre realtà europee.

- Nessi tra eredità architettoniche/topografiche e costruzione del discorso museale

- Impatto pubblico e possibile utenza

- Dimensione didattica e formativa

La redazione della rivista si riserva libertà di valutazione sull'accoglimento e la pubblicazione degli interventi ricevuti.

Il Comitato redazionale 